\title{
Aspartate Aminotransferase Antigen Measurement
}

National Cancer Institute

\section{Source}

National Cancer Institute. Aspartate Aminotransferase Antigen Measurement. NCI

Thesaurus. Code C81978.

The determination of the amount of aspartate aminotransferase antigen present in a sample. 